\title{
Escrubagem da bauxita de Paragominas - PA. Parte 1: influência de variáveis operacionais na desagregação da bauxita
}

\section{Scrubbing process of the Miltonia 3 Bauxite - Part 1: influence of operating variables on bauxite disintegration}

\section{Jaime Henrique Barbosa da Costa Engenheiro Químico, \\ Prof. MSc. da Coordenação de Mineração IFPA \\ jaime.costa@ifpa.edu.br}

\section{Homero Delboni Júnior}

Engenheiro de Minas,

Prof. Dr. do Departamento de Engenharia de Minas e Petróleo da Escola Politécnica da USP hdelboni@usp.br

\section{Resumo}

O objetivo desse trabalho foi investigar a influência de variáveis operacionais na geração de finos (material com granulometria menor que 0,037 $\mathrm{mm}$ ) durante o processo de escrubagem, em escala de laboratório, da bauxita proveniente da jazida Miltônia 3 da Mina de Bauxita de Paragominas-PA, com vistas a fornecer subsídios para a modelagem matemática da operação. Para isso, foi realizada uma campanha de experimentos de escrubagem, em um tambor desagregador de laboratório, baseada em planejamento fatorial. Os parâmetros operacionais avaliados foram os seguintes: grau de enchimento, tempo de residência da polpa e velocidade de rotação. A variável de resposta selecionada foi a quantidade de finos (partículas menores que $0,037 \mathrm{~mm}$ ) no produto desagregado. O programa de experimentos permitiu a análise da influência de cada variável operacional selecionada na desagregação da bauxita. De acordo com os resultados obtidos, o parâmetro operacional que produziu o efeito mais significativo, na variável de resposta, foi o grau de enchimento.

Palavras-chave: Escrubagem, bauxita, tambor desagregador.

\begin{abstract}
The aim of this study was to investigate the bauxite scrubbing process on samples from Miltonia 3, a Vale operation in the State of Pará, Brazil. The experimental program included the design of a standard laboratory test, from which parameters were derived for predicting the operation of a scrubber in steady state conditions. Three main variables were selected for the laboratory experimental program using the factorial design technique. These were load fraction, residence time and rotation speed. The amount of fines was determined through screening both the feed and the product of the scrubbing test. The former was considered to be a characteristic material, while the second was the dependent variable, i.e. the result of the scrubbing process. According to experiments, the load fraction was the most important variable for the scrubbing process.
\end{abstract}

Keywords: Scrubbing, bauxite, drum scrubber. 


\section{Introdução}

Taggart (1945) define a operação unitária de escrubagem de minérios como sendo a desagregação por meio de forças relativamente leves. Tais forças podem ser comparadas com os esforços usuais em cominuição, e devem ser suficientes para reduzir materiais razoavelmente moles e inconsolidados, tais como argilas. Também devem ser capazes de separar grãos unidos entre si por ligações brandas, geradas, por exemplo, na cimentação natural ocorrida com certos minérios ou na precipitação de sais.

Segundo Trajano (1966), por lavagem (washing) de minérios, entende-se a separação de dois constituintes desses minérios que difiram distintamente em granulometria. Na maioria das vezes, o constituinte mais fino, geralmente argila ou material argiloso, encontra-se mais ou menos aglomerado ou aderido ao constituinte grosseiro, que é quase sempre o mineral valioso, salvo casos especiais ou quando o beneficiamento é da própria argila. Nessas condições, a escrubagem

\section{Materiais e métodos}

\section{Materiais}

A amostra de bauxita foi fornecida pela VALE, para realização dos ensaios, e foi obtida a partir da pilha de homogeneização do circuito industrial da usina de beneficiamento compreende um estágio prévio, ou simultâneo à lavagem, de desagregação do minério com água, a fim de se individualizarem as micropartículas da argila e limpar as partículas grosseiras do outro mineral da argila aderida.

O equipamento utilizado para realizar tal processo é conhecido como scrubber. Existem diferentes equipamentos de escrubagem no mercado e o que se utilizou, nesse trabalho, foi o chamado drum scrubber ou tambor desagregador. $\mathrm{O}$ equipamento industrial consiste de um cilindro, aberto nas extremidades e ligeiramente inclinado em relação à horizontal, que gira em torno de seu próprio eixo, montado sobre rolos.

No Brasil, os drum scrubbers são utilizados na desagregação e lavagem de bauxitas em várias usinas de beneficiamento, como, por exemplo, Mineração Rio do Norte (MRN) em Trombetas, Alcoa em Juruti, ambas localizadas no Estado do Pará, e Companhia Brasileira de Alumínio (CBA) em Miraí e Itamarati- MG (Alves \& Reis, 2008).
Segundo Varela (2009), a principal justificativa para utilização de equipamentos de escrubagem e lavagem, em plantas de processamento de materiais, é a remoção de partículas finas. Estas partículas, normalmente, são consideradas uma fração indesejável, diminuindo o valor agregado do produto final, e encontram-se aderidas às partículas minerais de maior tamanho, sendo imprescindível a sua retirada do processo. Muitas vezes, essa contaminação por finos encontra-se na forma de aglomerados, que necessitam ser quebrados, dissolvidos e, por fim, separados da fração grossa.

O presente trabalho teve por objetivo investigar a influência das variáveis operacionais grau de enchimento, velocidade de rotação e tempo de residência, na geração de finos (material com granulometria menor que $0,037 \mathrm{~mm}$ ), durante o processo de escrubagem da bauxita, em escala de laboratório, com vistas a fornecer subsídios para a modelagem matemática da operação.

\section{Equipamentos}

Os equipamentos utilizados, nos ensaios de escrubagem (Figura 1), foram: da Mina de Bauxita de Paragominas (MBP). O minério utilizado, nos ensaios de escrubagem, foi o do platô Miltônia 3. O procedimento, para obtenção da amostra primária, consistiu

da retirada de incrementos dessa pilha, da formação de uma pilha alongada, para homogeneização dos incrementos, e da retirada da amostra final para trabalho.

um tambor metálico e um acionador com ajuste da velocidade de rotação.

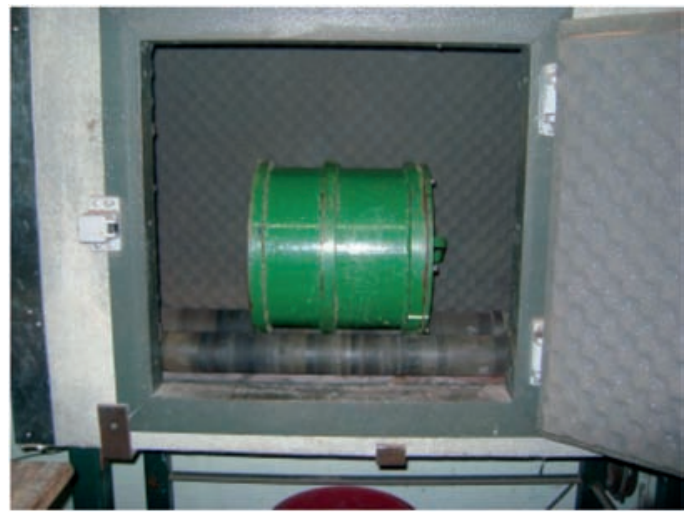

As características do tambor metálico são mostradas na Tabela 1.

Figura 1

Equipamento utilizado nos ensaios de escrubagem em escala de laboratório.

\section{Métodos}

Os ensaios de escrubagem, em escala de laboratório, tiveram, por objeti- vo, verificar a influência de variáveis operacionais no desempenho do processo.
As variáveis estudadas foram as seguintes: grau de enchimento $(\mathrm{Ge})$, tempo de 
residência da polpa $\left(t_{\mathrm{r}}\right)$ e velocidade de rotação $(\mathrm{Vr})$.

O procedimento experimental consistiu de peneiramento de toda a amostra na peneira de abertura de $76,2 \mathrm{~mm}$, britagem do material retido na malha de $76,2 \mathrm{~mm}$ até que todo produto passasse nesta malha, secagem em estufa a $100^{\circ} \mathrm{C}$, homogeneização e quarteamento, a fim de se obterem alíquotas para realização dos ensaios. As massas de sólidos, para os ensaios, foram de 813 g e 1626 g, determinadas para graus de enchimento de 5 e 10\%, respectivamente. A distribuição granulométrica da alimentação dos ensaios foi determinada através de peneira-

Tabela 1

Características do tambor utilizado nos ensaios de laboratório.

Tabela 2

Variáveis operacionais e seus respectivos níveis.

Tabela 3

Condições de realização dos ensaios de escrubagem em escala de laboratório. mento manual a úmido.

O programa de ensaios foi planejado de forma a permitir a análise da influência das variáveis operacionais do processo de escrubagem na desagregação da amostra de bauxita. O método adotado, para execução dos ensaios, foi baseado num planejamento fatorial $2^{3}$ (Barros Neto et al., 2001). Na Tabela 2, são apresentadas as variáveis selecionadas para o estudo e seus respectivos níveis de trabalho.

Os ensaios foram realizados em duplicata, em ordem aleatória e com concentração de sólidos da polpa de $50 \%$. As condições operacionais de cada en- saio são apresentadas na Tabela 3.

O parâmetro de resposta utilizado para avaliar a desagregação ocorrida durante os ensaios foi a porcentagem passante, em peneira de abertura $0,037 \mathrm{~mm}$ (400 \#), do produto desagregado, a qual foi denominada de finos no produto (FP). Nesse trabalho, consideraram-se como finos as partículas com tamanhos inferiores a 0,037 $\mathrm{mm}$, pois é abaixo dessa granulometria que se encontra o argilomineral caulinita, principal portador da sílica reativa, contaminante do minério, a qual deve ser individualizada e separada.

\begin{tabular}{|c|c|c|c|c|}
\hline \multicolumn{3}{|c|}{ Características } & \multicolumn{2}{|c|}{ Valores } \\
\hline \multicolumn{3}{|c|}{ Comprimento interno: } & \multicolumn{2}{|c|}{$305 \mathrm{~mm}$} \\
\hline \multicolumn{3}{|c|}{ Diâmetro interno: } & \multicolumn{2}{|c|}{$305 \mathrm{~mm}$} \\
\hline \multicolumn{3}{|c|}{ Relação de aspecto (L/D): } & \multicolumn{2}{|c|}{$1: 1$} \\
\hline \multicolumn{3}{|c|}{ Altura das aletas de revolvimento: } & \multicolumn{2}{|c|}{$6 \mathrm{~mm}$} \\
\hline \multicolumn{3}{|c|}{ Quantidade de aletas: } & \multicolumn{2}{|c|}{4} \\
\hline \multicolumn{3}{|c|}{ Volume do cilindro: } & \multicolumn{2}{|c|}{$22,3 \mathrm{~L}$} \\
\hline \multicolumn{3}{|c|}{ Velocidade crítica $\left(V_{c}\right)$ : } & \multicolumn{2}{|c|}{$76,6 \mathrm{rpm}$} \\
\hline \multicolumn{3}{|c|}{ Variáveis Operacionais } & \multicolumn{2}{|c|}{ Níveis } \\
\hline \multicolumn{3}{|c|}{ Grau de Enchimento - (\%) } & \multicolumn{2}{|c|}{5 e 10} \\
\hline \multicolumn{3}{|c|}{ Tempo de Residência da Polpa - (min) } & \multicolumn{2}{|c|}{1 e 3} \\
\hline \multicolumn{3}{|c|}{ Velocidade de Rotação - $(\% \vee c)$} & \multicolumn{2}{|c|}{28,4 e 41,4} \\
\hline Ordem & Ensaio & Ge (\%) & $\operatorname{tr}(\min )$ & $\mathrm{Vr}(\% \mathrm{Vc})$ \\
\hline 3 & 1 & 5 & 1 & 28,4 \\
\hline 4 & 2 & 10 & 1 & 28,4 \\
\hline 2 & 3 & 5 & 3 & 28,4 \\
\hline 1 & 4 & 10 & 3 & 28,4 \\
\hline 6 & 5 & 5 & 1 & 41,4 \\
\hline 8 & 6 & 10 & 1 & 41,4 \\
\hline 7 & 7 & 5 & 3 & 41,4 \\
\hline 5 & 8 & 10 & 3 & 41,4 \\
\hline
\end{tabular}

\section{Resultados e discussão}

\section{Distribuição granulométrica da alimentação dos ensaios}

A distribuição granulométrica da alimentação dos ensaios indicou que cerca de $90 \%$ das partícu- las possuem tamanho menores que $50,8 \mathrm{~mm}, 50 \%$ encontram-se abaixo de $9,53 \mathrm{~mm}$ e $23 \%$ estão abaixo de
0,037 $\mathrm{mm}$, conforme pode ser observado na Figura 2.

\section{Influência das variáveis operacionais na geração de finos}

O objetivo desse item foi determinar quais dos efeitos das variáveis operacionais dos ensaios foram significativos na desagregação da bauxita. Nessa etapa, considerou-se, como geração de finos (GF), o acréscimo da quantidade de material, obtido após os ensaios, com granulometria menor que $0,037 \mathrm{~mm}(400$ \#). Os resultados dos ensaios de escrubagem em laboratório, com base no plane- jamento fatorial $2^{3}$, para estudar o efeito do grau de enchimento, tempo de residência de polpa e velocidade de rotação sobre a geração de finos, são mostrados na Tabela 4.

Nos planejamentos fatoriais de dois níveis, identificam-se os níveis, superior e inferior, com os sinais (+) e (-), respectivamente (Tabela 4).

Os resultados dos efeitos das vari- áveis operacionais, na resposta da operação de escrubagem da bauxita, em escala de laboratório, são observados na Tabela 5.

Utilizou-se o teste $t$ de Student, com $90 \%$ de confiança e 8 graus de liberdade, para decidir quais dos efeitos dos fatores foram significativos na resposta selecionada, nesse nível de confiança. Aplicando esse critério aos valores da Tabela 5 


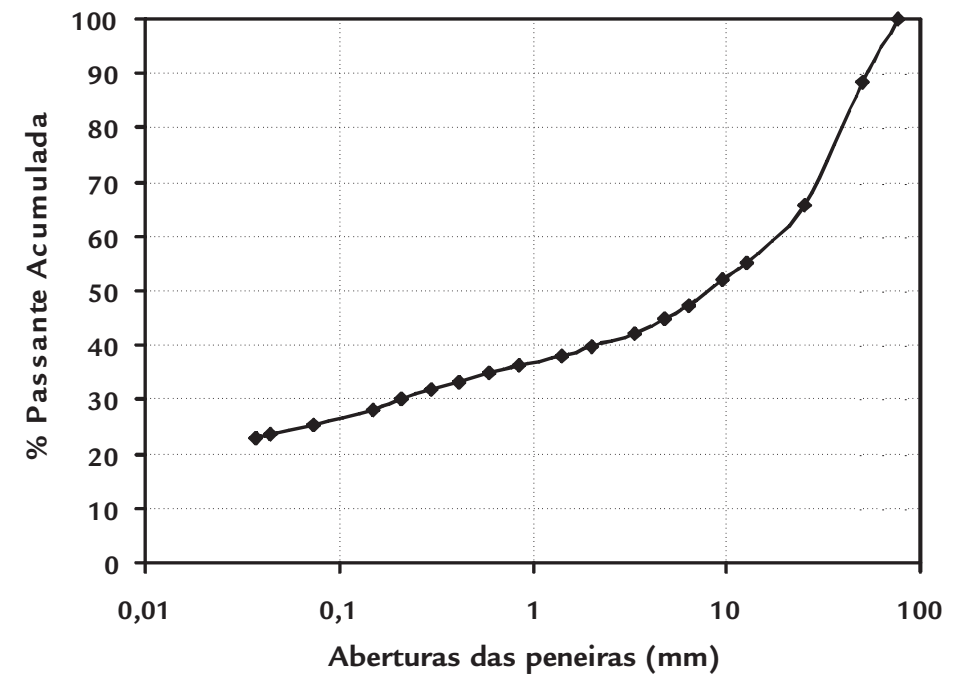

\begin{tabular}{c|c|c|c|c|c|c}
\hline \multirow{2}{*}{ Ensaio } & \multicolumn{3}{|c|}{ Fatores } & \multicolumn{2}{c|}{ Resposta } & \multirow{2}{*}{ Média } \\
\cline { 2 - 6 } & $\mathbf{1}$ & $\mathbf{2}$ & $\mathbf{3}$ & \multicolumn{2}{c}{ (\% passante em 0,037 $\mathbf{m m}$ ) } & \\
\hline 1 & - & - & - & 23,5 & 28,5 & 26,0 \\
\hline 2 & + & - & - & 33,6 & 24,0 & 28,8 \\
\hline 3 & - & + & - & 28,1 & 27,7 & 27,9 \\
\hline 4 & + & + & - & 32,5 & 29,6 & 31,1 \\
\hline 5 & - & - & + & 28,8 & 28,1 & 28,5 \\
\hline 6 & + & - & + & 29,1 & 37,3 & 33,2 \\
\hline 7 & - & + & + & 29,0 & 30,1 & 29,6 \\
\hline 8 & + & + & + & 31,0 & 37,2 & 34,1 \\
\hline
\end{tabular}

\begin{tabular}{c|c|c}
\hline Fatores & $(-)$ & $(+)$ \\
\hline 1: Grau de Enchimento - $(\%)$ & 5 & 10 \\
\hline 2: Tempo de Residência da Polpa - (min) & 1 & 3 \\
\hline 3: Velocidade de Rotação - $(\% \mathrm{Vc})$ & 28,4 & 41,4 \\
\hline Resposta & \multicolumn{2}{|c}{ Finos no produto - $\%$ passante em $0,037 \mathrm{~mm})$} \\
\hline
\end{tabular}

\begin{tabular}{|c|c|}
\hline Média de Finos no Produto: & $29,9 \pm 0,95$ \\
\hline \multicolumn{2}{|l|}{ Efeitos principais: } \\
\hline 1 (Grau de Enchimento) & $3,81 \pm 1,91$ \\
\hline 2 (Tempo de Residência de Polpa) & $1,54 \pm 1,91$ \\
\hline 3 (Velocidade de Rotação) & $2,89 \pm 1,91$ \\
\hline \multicolumn{2}{|l|}{ Interação de dois fatores: } \\
\hline 12 & $0,04 \pm 1,91$ \\
\hline 13 & $0,84 \pm 1,91$ \\
\hline 23 & $-0,54 \pm 1,91$ \\
\hline \multicolumn{2}{|l|}{ Interação de três fatores: } \\
\hline 123 & $-0,14 \pm 1,91$ \\
\hline
\end{tabular}

observou-se que somente o efeito do grau de enchimento foi significativo.

Não há evidência de interação do grau de enchimento com os outros dois fatores, nesse nível de confiança, conforme pode ser observado na Tabela 5 pois os valores das interações estão próximos de zero.

Os menores valores de finos no produto $(26,0 \%$, em média) foram obtidos

\section{Conclusões}

De acordo com os resultados dos ensaios experimentais, as principais con-
Figura 2

Distribuição granulométrica da alimentação dos ensaios de escrubagem.

Tabela 4

Matriz de planejamento.

\section{Tabela 5}

Resultados dos efeitos para o planejamento fatorial $2^{3}$ dos ensaios de escrubagem em laboratório.

quantidade de finos no produto. Não há evidência de que esse aumento dependa dos níveis das outras variáveis, na faixa experimental investigada.

Pode-se observar, na Figura 3, que, aumentando o grau de enchimento, aumenta-se a quantidade de finos no produto, mas o efeito é mais pronunciado com a velocidade de rotação de $41,4 \%$ Vc do que com a de $28,4 \%$ Vc.
Entre os parâmetros operacionais estudados, em escala de laboratório, o 
grau de enchimento foi o que produziu efeito estatisticamente mais significativo, no nível de $90 \%$ de confiança, na desagregação da bauxita.

Não se verificou efeito de interação

Figura 3

Efeito do grau de enchimento na quantidade de finos no produto.

\section{Agradecimentos}

Os autores agradecem ao IFPA pela liberação do professor para realização da estatisticamente significativo na desagregação da bauxita, no nível de 90\% de confiança, entre os parâmetros operacionais estudados na faixa experimental investigada.
Os parâmetros tempo de residência da polpa e velocidade de rotação não apresentaram efeitos estatisticamente significativos na desagregação da bauxita, no nível de $90 \%$ de confiança.

\section{Referências bibliográficas}

pós-graduação em engenharia mineral, à CAPES pela concessão da bolsa de Dou-

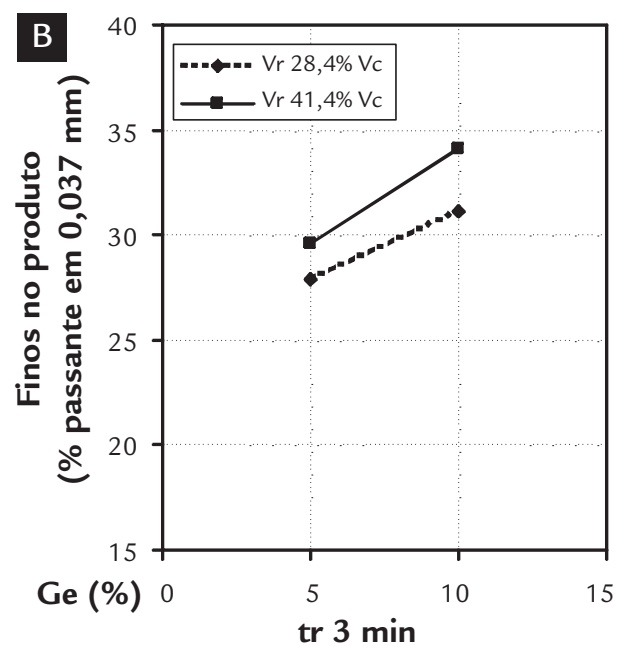

torado e à VALE pelo fornecimento das amostras.

ALVES, F. E., REIS, R. L. G. Miraí, a mais nova unidade de bauxita da CBA já opera em ritmo quase normal. Brasil Mineral, São Paulo, n. 276, p. 26-29, agosto 2008.

BARROS NETO, B. et al. Como fazer experimentos: pesquisa e desenvolvimento na ciência e na indústria. Campinas: Editora da Unicamp, 2001. 401 p.

TAGGART, A.F. Handbook of mineral dressing. New York, USA: John Wiley \& Sons, 1945. v.1, p.10-01 a 10-16.

TRAJANO, R. B. Princípios de tratamento de minérios: operações unitárias e aparelhos. Rio de Janeiro, 1966. p. 62-63.

VARELA, J. J. Estado da arte do processo de lavagem de minérios: conceito, aplicação e desenvolvimento. In: ENCONTRO NACIONAL DE TRATAMENTO DE MINÉRIOS E METALURGIA EXTRATIVA, 23. Anais... Porto alegre: Gramado, 2009. v. 1. p. 225- 232.

Artigo recebido em 15 de março de 2010. Aprovado em 10 outubro de 2011. 